\title{
Preoperative prognostic nutritional index shows no significant prognostic value for short-term outcomes of anastomosis-leakage patients after cancerous esophagectomy
}

\author{
Gu-Ha A-Lai ${ }^{1 \#}$, Han-Yu Deng, ${ }^{1,2 \#}$, Tie-Niu Song ${ }^{1}$, Jun Luo ${ }^{1}$, Ze-Guo Zhuo ${ }^{1}, X_{\text {u Shen }}{ }^{1}$, Yi-Dan Lin ${ }^{1}$ \\ ${ }^{1}$ Department of Thoracic Surgery, ${ }^{2}$ Lung Cancer Center, West China Hospital, Sichuan University, Chengdu 610041, China \\ Contributions: (I) Conception and design: YD Lin, GH A-Lai; (II) Administrative support: YD Lin; (III) Provision of study materials or patients: GH \\ A-Lai; (IV) Collection and assembly of data: J Luo, ZG Zhuo, X Shen; (V) Data analysis and interpretation: HY Deng, TN Song; (VI) Manuscript \\ writing: All authors; (VII) Final approval of manuscript: All authors. \\ \#These authors contributed equally to this work. \\ Correspondence to: Yi-Dan Lin. Department of Thoracic Surgery, West China Hospital, Sichuan University, No. 37 Guoxue Alley, Chengdu 610041, \\ China. Email: linyidan.academy@foxmail.com.
}

Background: The relationship between preoperative nutritional and immunological status and long-term outcome after cancerous esophagectomy has been investigated widely. Growing evidence also demonstrated preoperative nutritional and immunological status also affects short-term outcome after surgery for esophageal cancer. However, the relationship between preoperative nutritional and immunological status and short-term outcome of anastomosis-leakage patients after cancerous esophagectomy was scarce. The aim of this study was to evaluate the association between preoperative prognostic nutritional index (PNI) and shortterm outcome of anastomosis-leakage patients after surgery.

Methods: In this study, we retrospectively enrolled 90 patients who were confirmed to be esophageal cancer by preoperative biopsy or postoperative pathological review and also suffered postoperative anastomotic leakage from January 2014 to June 2017 at the Department of Thoracic Surgery, West China Hospital. Then we evaluated the association between PNI and short-term surgical outcome. The endpoints included postoperative mortality, postoperative hospital duration, postoperative intensive care unit (ICU) duration, hospitalization cost.

Results: The cut-off value of PNI was set at 49.83 in our study, patients with a preoperative PNI $\geq 49.83$ were divided into high-PNI group, while those with a preoperative PNI $<49.83$ were classified into low-PNI group. For the postoperative anastomosis-leakage patients in the two groups, baseline characteristics were all comparable, and analysis revealed no significantly statistical difference between the two groups regarding mortality, postoperative hospital duration and postoperative ICU duration. Though mean hospital-duration cost $(144,791.08 \pm 87,312.87$ vs. $127,364.25 \pm 69,233.16)$ was more in the low-PNI group, there was still no significant difference demonstrated $(\mathrm{P}=0.297)$. There was no significant difference revealed between the subgroups of non-death patients from the two original groups concerning the endpoints, while the hospitalduration cost of the high-PNI group tended to be lower than low-PNI group $(125,262.80 \pm 71,304.12$ vs.

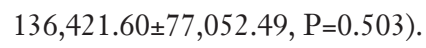

Conclusions: Although in-hospital cost of high-PNI group tended to be lower than low-PNI group, preoperative PNI showed no significant prognostic value for short-outcomes of anastomosis-leakage patients after cancerous esophagectomy. More prospective studies were badly needed to provide more evidence in the future.

Keywords: Prognostic nutritional index (PNI); cancerous esophagectomy; anastomosis leakage; short outcome

Submitted Sep 08, 2019. Accepted for publication Nov 05, 2019.

doi: 10.21037/apm.2019.11.08

View this article at: http://dx.doi.org/10.21037/apm.2019.11.08 


\section{Introduction}

Esophageal cancer is the eighth most common cancer and one of the most aggressive malignancies which resulted in sixth cancer related death worldwide, the 5 -year survival is just around $15-25 \%$ (1). However, as for the patients who underwent curative cancer surgery, the 5 -year overall survival is significantly improved which increases to $40 \%$ (2). Owing to continuous improvements in surgery procedures and related auxiliary therapies, either short-term or long-term prognosis has been significantly improved in recent years (2-5). However, postoperative complications especially anastomosis leakage and other life-threatening complications still badly troubled surgeons, that results in unsatisfactory prognosis and giant economic burden (6-8).

Preoperative prognostic nutritional index (PNI) was originally developed to predict risk of perioperative and risk of postoperative morbidity and mortality for gastrointestinal surgery $(9,10)$. The PNI which was simplified by Onodera et al. was defined based on the serum albumin level and peripheral blood lymphocyte count (11). PNI which directly represents the nutritional and immunological status of patients was usually low in esophageal cancer patients, resulting from difficulty in eating and chronic cost of the tumor. Increasing studies demonstrated that preoperative immunologic and nutritional status associated with either postoperative complications or long-term outcomes of cancer patients (12-14). With regard to esophageal cancer, it was showed that PNI was a significant and independent predictor of long-term outcomes of patients who received curative esophagectomy or neoadjuvant chemotherapy, and PNI could act as a marker of survival $(10,15,16)$. What's more, recent studies also proved that preoperative PNI could provide predictive information for postoperative complications in patients with esophageal carcinoma $(17,18)$.

On basis of the previous studies which demonstrated that PNI could be an independent predictor for both short-term and long-term outcomes of patients with esophagectomy. It is still unknown whether PNI could accurately predict the prognosis of anastomosis-leakage patients who were most concerned by surgeons. Thus, we hypothesize that preoperative PNI could provide predictive value for the anastomosis-leakage patients. Aiming to investigate the predictive value of preoperative PNI on the short-term outcomes of the patients suffered anastomotic leakage, including death rate, reoperation rate, costs and hospital duration.

\section{Methods}

For this retrospective observational study, a total of 97 consecutive patients who were diagnosed anastomosis leakage after undergoing curative esophagectomy and lymphadenectomy were retrieved from January 2014 to June 2017 in West China Hospital. Tumor stage of the patients were defined according to the seventh edition of the American Joint Committee on Cancer TNM classification system. The eligibility criteria for review were as follows: (I) underwent esophagectomy and regional lymphadenectomy (including abdominal and thoracic lymph nodes dissection, cervical lymph nodes of some patients were also dissected); (II) esophageal cancer proven by pathology; (III) underwent R0 resection regarding the surgical margin; (IV) no distant metastasis (M1) before surgery; (V) anastomotic leakage confirmed by endoscopy or methylene blue test postoperatively. The exclusion criteria were as follows: (I) suffered acute or chronic inflammatory disease; (II) diagnosed hematological or autoimmune disease; (III) with clinical data missing; (IV) suffered other malignancies other than esophageal cancer. All those patients received endoscopy, endoscopic ultrasound, chest computed tomography (CT), abdominal CT, cervical ultrasonography, and pulmonary function and blood testing routinely, evaluating as resectable esophageal cancer preoperatively. All the patients received open or minimally invasive esophagectomy and systematic lymph nodes dissection, surgery procedure included Ivor-Lewis or Sweet or McKeown procedure. Because this study was a retrospective prognostic analysis and analyzed anonymously, the Ethics Committee of West China Hospital, Sichuan University waived the need for informed consents from those patients, while an approval for the ethical committee was obtained (approval number: 20191022).

The end point parameters including secondary surgery, in-hospital mortality after anastomotic leakage, postoperative hospital duration, postoperative intensive care unit (ICU) duration and in-hospital cost. The clinical and pathological data including gender, age, smoking history, alcohol history, tumor location, tumor size, histological type, lymph node metastasis, TNM stage, and preoperative routine laboratory data including routine blood test and the hepatic function test before breakfast within 2 weeks before surgery were collected. The definitions of PNI was calculated as follows: $\mathrm{PNI}=10 \times$ serum albumin $(\mathrm{g} / \mathrm{dL})$ $+0.005 \times$ total lymphocyte count (per mm3) (11). 


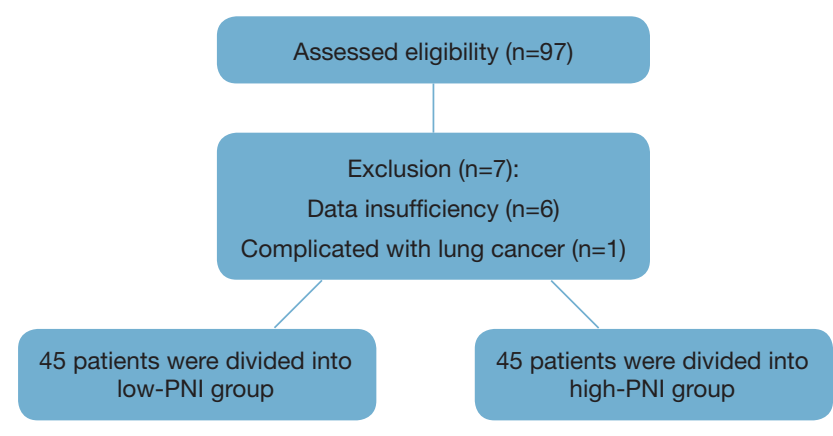

Figure 1 Flow chart showing patient recruitment. PNI, prognostic nutritional index.

One-sample Kolmogorov-Smirnov test

\begin{tabular}{|c|c|c|}
\hline \multicolumn{2}{|c|}{} & PNI \\
\hline \multirow{2}{*}{ Normal Parameters ${ }^{\mathrm{a}, \mathrm{b}}$} & Mean & 90 \\
\cline { 2 - 3 } & Std. deviation & 50.0650 \\
\hline \multirow{2}{*}{ Most extreme differences } & Absolute & 0.112 \\
\cline { 2 - 3 } & Positive & 0.112 \\
\cline { 2 - 3 } & Negative & -0.067 \\
\hline \multicolumn{2}{|c|}{ Test statistic } & 0.112 \\
\hline \multicolumn{2}{|c|}{ Asymp. Sig. (two-tailed) } & $0.007^{\circ}$ \\
\hline
\end{tabular}

Figure 2 Normal distribution test of PNI. a , test distribution is normal; ${ }^{\text {b }}$, calculated from data; ${ }^{\text {c }}$, Lilliefors significance correction. PNI, prognostic nutritional index.

\section{Statistical analysis}

All analyses were performed using Statistical Package for Social Science (SPSS for Windows, version 22.0, SPSS Inc., Chicago, IL, USA) program. Subject characteristics were compared using Chi-squared test or Fisher exact test for categorical variables and Student's t-test or Mann-Whitney $\mathrm{U}$ test for continuous variables. Kolmogorov-Smirnov test was applied to examine characteristics of PNI distribution. A $P$ value of less than 0.05 was considered significant.

\section{Results}

A total of 97 patients with confirmed anastomotic leakage after cancerous esophagectomy were enrolled in this study primarily, preoperative PNI of three patients could not be gained owing to data of the hepatic function test was missing, postoperative pathological reports of three patients were missing, and diagnosis of 1 patient combined with lung cancer, then 90 patients were included in this study for analysis finally as demonstrated in Figure 1. Mean age of all the patients were 64.34 years old, the mean PNI value was 50.10, while the median value of PNI was 49.83 .

As shown in Figure 2, characteristics of distribution of the PNI was skewed distribution examined by KolmogorovSmirnov test $(\mathrm{P}=0.007)$, we extracted the median value of the PNI values as cut-off value. The cut-off value of PNI was set at 49.83 in our study, then 45 patients with a preoperative $\mathrm{PNI} \geq 49.83$ were divided into high-PNI group, while another 45 patients with a preoperative PNI $<49.83$ were classified into low-PNI group.

With regard to relationship between different variables and PNI, analysis demonstrated that a strong positive correlation was detected between the level of albumin and lymphocyte count and PNI $(\mathrm{P}<0.001)$. Besides, it seemed that the preoperative PNI tended to be increased in low hospital-duration cost group compared with high hospital-duration cost group $(51.00 \pm 5.20$ vs. $49.13 \pm 4.89)$, no significant difference was observed $(\mathrm{P}=0.083)$. No significantly statistical differences of PNI were detected grouping by other variables as showed in Table 1 . The demographics, pathological and other baseline characteristics of all the 90 patients included in this study were demonstrated in Table 2. Tumor size of the lowPNI group was significantly larger than high-PNI group which was measured by the longest diameter $(4.73 \pm 2.30$ vs. 3.88 $\pm 1.69, \mathrm{P}=0.047)$. Compared with low-PNI group, the level of albumin, lymphocyte count and PNI were both statistically significant higher in high-PNI group $(\mathrm{P}<0.001)$. While all the other baseline characteristics were comparable between the two groups. Comparison of the endpoints between low and high-PNI groups was showed in Table 3. Postoperative hospital duration, mortality in hospital and post-leakage hospital duration were all comparable between two groups. The reoperation ratio seemed higher in low-PNI group, while no significant difference was observed $(\mathrm{P}=0.266)$. What's more, the hospital-duration cost in high-PNI group inclined to be lower than in low-PNI group, even though it was statistically similar $(127,364.25 \pm 69,233.16$ vs. $144,791.08 \pm 87,312.87, \mathrm{P}=0.297)$.

There were five hospital-duration deaths after anastomosis leakage in two groups respectively. Then subgroup analysis of the non-death patients in the two groups were conducted. As demonstrated in Table 4, the level of preoperative PNI, albumin and lymphocyte count 
Table 1 Relationship between kinds of variables and the PNI

\begin{tabular}{|c|c|c|c|}
\hline Variables & $\mathrm{N}$ & PNI & $P$ value \\
\hline Age (years old) & & & 0.274 \\
\hline$\leq 65$ & 47 & $50.63 \pm 4.66$ & \\
\hline$>65$ & 43 & $49.45 \pm 5.54$ & \\
\hline Sex & & & 0.295 \\
\hline Male & 71 & $49.77 \pm 5.29$ & \\
\hline Female & 19 & $51.16 \pm 4.29$ & \\
\hline Alcohol & & & 0.743 \\
\hline Yes & 41 & $49.87 \pm 4.53$ & \\
\hline No & 49 & $50.23 \pm 5.58$ & \\
\hline Smoking & & & 0.405 \\
\hline Yes & 60 & $50.38 \pm 5.26$ & \\
\hline No & 30 & $49.43 \pm 4.81$ & \\
\hline Albumin (g/L) & & & $<0.001$ \\
\hline$\leq 40$ & 30 & $45.49 \pm 3.33$ & \\
\hline$>40$ & 60 & $52.35 \pm 4.23$ & \\
\hline Lymphocyte count $\left(10^{9} / \mathrm{L}\right)$ & & & $<0.001$ \\
\hline$\leq 1.64$ & 45 & $47.43 \pm 4.12$ & \\
\hline$>1.64$ & 45 & $52.70 \pm 4.65$ & \\
\hline Tumor depth & & & 0.544 \\
\hline $\mathrm{T} 1-\mathrm{T} 2$ & 35 & $50.48 \pm 5.43$ & \\
\hline T3-T4 & 55 & $49.80 \pm 4.92$ & \\
\hline Lymph node metastasis & & & 0.775 \\
\hline Positive & 38 & $50.25 \pm 5.69$ & \\
\hline Negative & 52 & $49.93 \pm 4.69$ & \\
\hline Pathological stage & & & 0.771 \\
\hline$|-| \mid$ & 46 & $50.22 \pm 5.16$ & \\
\hline III & 44 & $49.90 \pm 5.10$ & \\
\hline Differentiation degree & & & 0.098 \\
\hline G1-G2 & 52 & $49.30 \pm 4.83$ & \\
\hline G3 & 38 & $51.11 \pm 5.35$ & \\
\hline Comorbidity & & & 0.877 \\
\hline Yes & 50 & $50.14 \pm 4.77$ & \\
\hline No & 40 & $49.97 \pm 5.56$ & \\
\hline Cost (RMB) & & & 0.083 \\
\hline$\leq 115,600$ & 45 & $51.00 \pm 5.20$ & \\
\hline$>115,600$ & 45 & $49.13 \pm 4.89$ & \\
\hline \multicolumn{3}{|c|}{ Postoperative hospital duration (days) } & 0.755 \\
\hline$\leq 39$ & 45 & $50.23 \pm 4.78$ & \\
\hline$>39$ & 45 & $49.89 \pm 5.47$ & \\
\hline
\end{tabular}

Table 2 Baseline characteristics of the two groups

\begin{tabular}{|c|c|c|c|}
\hline Variables & Low-PNI group & High-PNI group & $P$ value \\
\hline Age (years old) & $65.38 \pm 9.45$ & $63.31 \pm 8.57$ & 0.280 \\
\hline Sex & & & 0.438 \\
\hline Male & 37 & 34 & \\
\hline Female & 8 & 11 & \\
\hline Alcohol & & & 0.832 \\
\hline Yes & 21 & 20 & \\
\hline No & 24 & 25 & \\
\hline Smoking & & & 0.655 \\
\hline Yes & 29 & 31 & \\
\hline No & 16 & 14 & \\
\hline Albumin (g/L) & $39.15 \pm 2.57$ & $43.83 \pm 2.81$ & $<0.001$ \\
\hline $\begin{array}{l}\text { Lymphocyte counts } \\
\left(10^{9} / \mathrm{L}\right)\end{array}$ & $1.41 \pm 0.40$ & $1.97 \pm 0.54$ & $<0.001$ \\
\hline PNI & $46.20 \pm 2.95$ & $53.93 \pm 3.67$ & $<0.001$ \\
\hline Neoadjuvant therapy & & & - \\
\hline Yes & 2 & 0 & \\
\hline No & 43 & 45 & \\
\hline Surgery methods & & & 0.833 \\
\hline MIE & 23 & 24 & \\
\hline OE & 22 & 21 & \\
\hline Surgery procedure & & & 0.378 \\
\hline Sweet & 11 & 16 & \\
\hline Ivor-Lewis & 10 & 6 & \\
\hline McKeown & 24 & 23 & \\
\hline \multicolumn{2}{|c|}{ Digestive tract reconstruction } & & 0.434 \\
\hline Stomach & 43 & 40 & \\
\hline Non-stomach & 2 & 5 & \\
\hline Anastomotic methods & & & 0.673 \\
\hline Sewn by hand & 22 & 24 & \\
\hline Sewn by machine & 23 & 21 & \\
\hline Anastomotic site & & & 0.288 \\
\hline Neck & 28 & 23 & \\
\hline Thorax & 17 & 22 & \\
\hline Bleeding $(\mathrm{mL})$ & $170.22 \pm 132.52$ & $179.89 \pm 108.54$ & 0.706 \\
\hline
\end{tabular}

Table 2 (continued)

PNI, prognostic nutritional index; RMB, renminbi. 
Table 2 (continued)

\begin{tabular}{|c|c|c|c|}
\hline Variables & Low-PNI group & High-PNI group & $P$ value \\
\hline Tumor site & & & 0.500 \\
\hline Upper & 6 & 7 & \\
\hline Medium & 24 & 28 & \\
\hline Lower & 15 & 10 & \\
\hline Tumor size $(\mathrm{cm})$ & $4.73 \pm 2.30$ & $3.88 \pm 1.69$ & 0.047 \\
\hline Tumor depth & & & 0.123 \\
\hline $\mathrm{T} 1$ & 7 & 12 & \\
\hline T2 & 11 & 5 & \\
\hline T3 & 11 & 17 & \\
\hline $\mathrm{T} 4$ & 16 & 11 & \\
\hline Total lymph nodes & $20.47 \pm 8.68$ & $16.64 \pm 9.15$ & 0.450 \\
\hline Positive lymph nodes & $1.22 \pm 2.49$ & $1.78 \pm 3.80$ & 0.414 \\
\hline Pathological stage & & & 0.200 \\
\hline I & 7 & 12 & \\
\hline II & 17 & 10 & \\
\hline III & 21 & 23 & \\
\hline $\begin{array}{l}\text { Lymph node } \\
\text { metastasis }\end{array}$ & & & 0.284 \\
\hline Positive & 16 & 21 & \\
\hline Negative & 29 & 24 & \\
\hline Lymph node stage & & & 0.529 \\
\hline No & 29 & 23 & \\
\hline N1 & 8 & 13 & \\
\hline N2 & 6 & 6 & \\
\hline N3 & 2 & 3 & \\
\hline Differentiation degree & & & 0.859 \\
\hline G1 & 3 & 5 & \\
\hline G2 & 23 & 21 & \\
\hline G3 & 19 & 19 & \\
\hline
\end{tabular}

PNI, prognostic nutritional index; RMB, renminbi; MIE, minimally invasive esophagectomy; OE, open esophagectomy.

were significant lower in the low-PNI group than in highPNI group $(\mathrm{P}<0.001)$. Besides, tumor size of the low-PNI group tended to be larger than high-PNI group, while it was not statistically significant $(4.64 \pm 2.32$ vs. $3.76 \pm 1.72$, $\mathrm{P}=0.059)$. Other demographics and characteristics were all similar between the two groups in the subgroup analysis. Concerning the endpoints analysis between the subgroups illustrated in Table 5, reoperation ratio, postoperative hospital duration and post-leakage hospital duration were all comparable between the two groups in subgroup analysis. However, the mean in-hospital cost in high-PNI group tended to be lower than in low-PNI group more than $10,000 \mathrm{RMB}$, even though no significant differences was

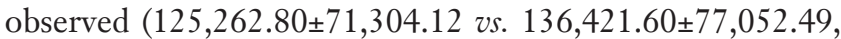
$\mathrm{P}=0.503)$.

\section{Discussion}

To our knowledge, there have been a large quantity of studies exploring the relationship between preoperative PNI and long or short outcomes of patients undergoing esophagectomy for esophageal cancer $(15,18)$, while this was the first study to investigate the prognostic value of preoperative PNI on short-term outcomes of anastomosisleakage patients after receiving cancerous esophagectomy. As for anastomosis-leakage patients after undergoing cancerous esophagectomy, this study found there were no significant differences between high preoperative PNI group and low preoperative PNI group concerning shortterm outcomes. Other than hospital-duration cost seemed to be higher in low preoperative PNI group both in original analysis and subgroup analysis, while it demonstrated no statistical significance. Besides, analysis revealed that tumor size of high-PNI group were significant smaller than in low-PNI group.

The PNI calculated from albumin and total lymphocyte count was initially designed to assess the nutritional and immunological status of patients undergoing surgery for gastrointestinal cancer (11). The cut-off value of preoperative PNI was defined to be 49.83 after analysis and calculation in this study, it was extracted from the median value of all the patients close to the mean value 50.065 after normal test. However, what's worth mentioning is that even though there have been a large number of studies relating to PNI, the cutoff value is still not reaching a consensus. Hirahara et al. in 2017 reported a cut-off value of 49.2 which was close to ours using receiver operating characteristics (ROC) curve and came to the conclusion that the PNI was a significant and independent predictor of cancer-specific survival and overall survival of esophageal squamous cell carcinoma (ESCC) patients after curative esophagectomy (10). In a study concerning the prognostic value of PNI on postoperative complications and survival in patients with resection of 
Table 3 endpoints of the two groups

\begin{tabular}{lcc}
\hline Variables & Low-PNI group & High-PNI group \\
\hline Reoperation & 6 & 2 \\
Yes & 39 & 43 \\
No & $41.78 \pm 21.58$ & $41.96 \pm 21.99$ \\
Postoperative hospital duration (days) & $33.31 \pm 20.76$ & $31.96 \pm 22.74$ \\
Post-leakage hospital duration (days) & & 0.969 \\
Death & 5 & 5.768 \\
Yes & 40 & 1.000 \\
No & $144,791.08 \pm 87,312.87$ & 40 \\
In-hospital cost (RMB) & & $127,364.25 \pm 69,233.16$ \\
\hline
\end{tabular}

$\mathrm{PNI}$, prognostic nutritional index; RMB, renminbi.

colorectal cancer, Mohri et al. also defined a PNI value of $\geq 50$ was locating in normal range (19). However, there still were other different cut-off values of PNI ranging from 45 to 55 (17,20-22). More robust trials and evidence are urgently performed to determine the accurate cut-off value of preoperative PNI in the future.

Our study demonstrated that the relationship between preoperative PNI and tumor size was negative. Similarly, previous studies have found enlarged tumor size, and higher TNM staging with preoperative PNI decreasing $(17,19,23)$, which indicated low-PNI usually related with more progressive tumor. As for the short-term outcomes, Filip et al. showed that preoperative PNI acted as an independent risk factor for predicting major complications (grade III-V of Clavien-Dindo classification) after cancerous esophagectomy (18). Other studies also demonstrated that preoperative PNI represented a useful indicator of the occurrence of complications and length of hospital stay, and may influence overall survival at 6 months after surgery, and in which the result of high-PNI group were more favorable $(17,24)$. On the contrary, Han-Geurts et al. concluded that preoperative nutritional parameters including PNI, Nutritional Risk Index (NRI), body mass index (BMI) and weight loss had no significant predictive value on postoperative complications in patients undergoing resection of esophageal cancer (25), which was analogous to this finding. Many studies have demonstrated that PNI was an independent prognostic factor for overall survival and advocated to act as a new maker of survival owing to costeffective and readily available $(10,26,27)$. Besides, the same result was found that high-PNI also act as an independent factor to predict better short- and long-term outcomes of gastric malignancy patients after surgery $(28,29)$. Oshi et al. also reported patients with low preoperative PNI tended to suffer a higher risk for anastomotic leakage after laparoscopyassisted total gastrectomy in 2016 (30). Concerning rectal cancer, Noh et al. concluded patients with anastomosis leakage were associated with poorer disease-free survival, and these anastomosis-leakage patients had a higher probability to suffer tumor recurrence while the PNI was decreasing to below 36 (31). What's interesting was increasing studies have demonstrated that systemic inflammation score and extended inflammation related factors were found to be novel and useful prognostic score for esophageal cancer patients after surgery, the prognosis was worsening with status of systemic inflammation decreasing (32-34). Absolutely, large, prospective studies of relationship between status of systemic inflammation and prognosis of esophageal patients are urgently needed in the future.

This study had several limitations. First, it was a retrospective study in nature which could limit the validity of our results. Second, this study still suffered from the limitation of 90 small sample size. Third, comparisons of long-term outcomes between high and low-PNI groups are badly needed for the patients with anastomosis leakage in the future. Finally, the adequacy of the cut-off value for the PNI was needed to be assessed.

\section{Conclusions}

In this retrospective study, it concluded that preoperative immunological and nutritional status established by PNI 
Table 4 Subgroup analysis of baseline characteristics of non-death patients

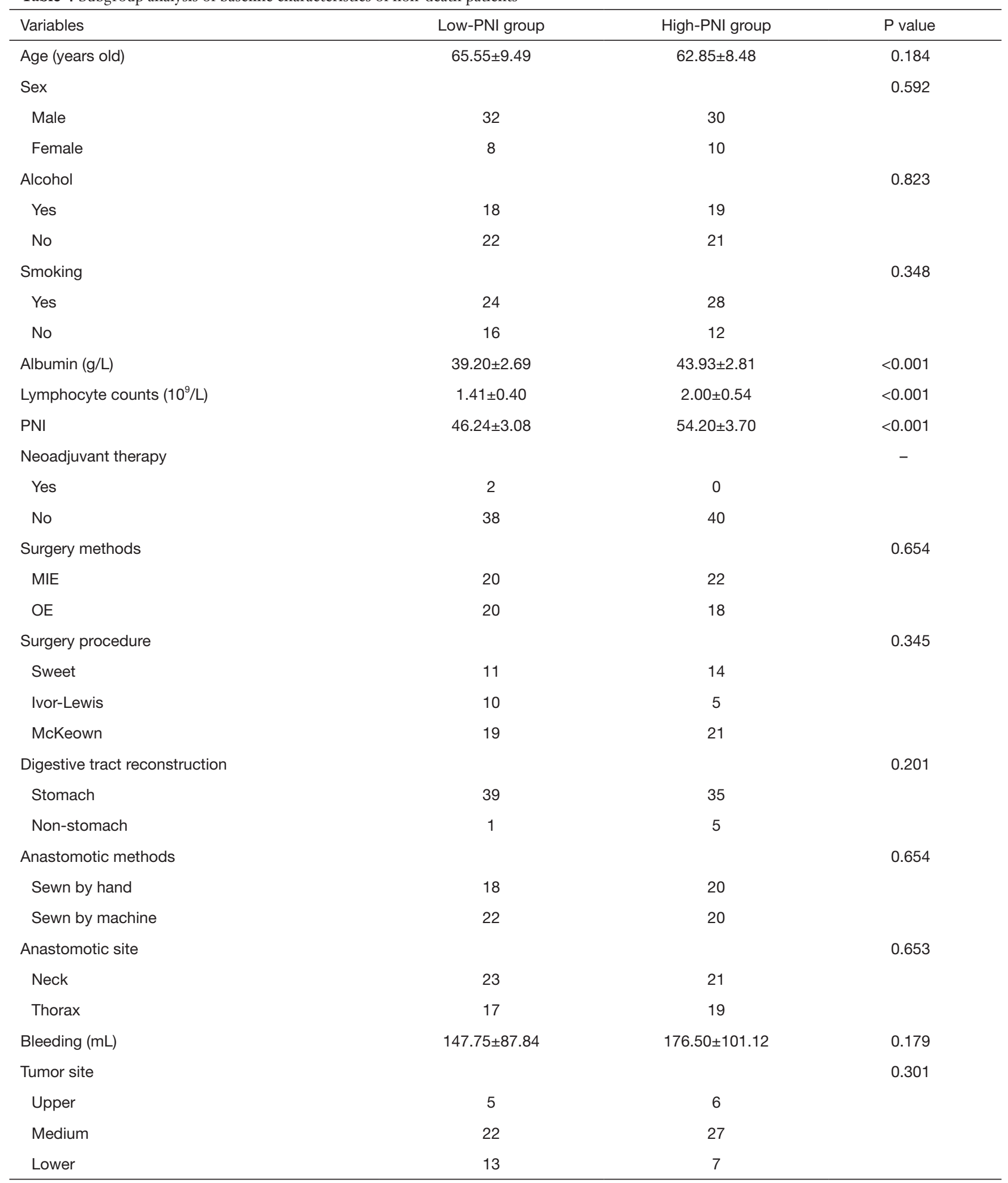

Table 4 (continued) 
Table 4 (continued)

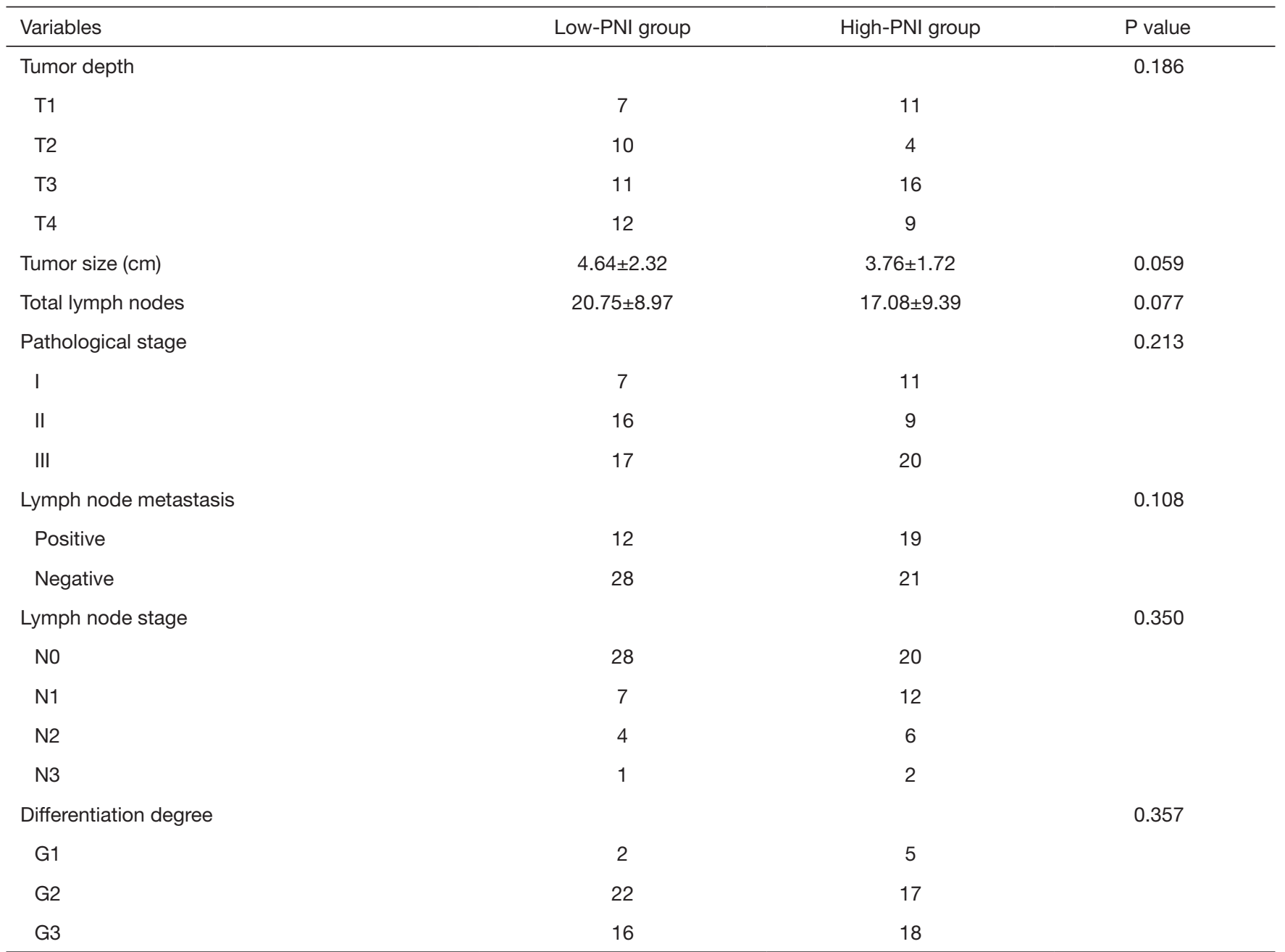

PNI, prognostic nutritional index; RMB, Renminbi; MIE, minimally invasive esophagectomy; OE, open esophagectomy.

Table 5 Subgroup analysis of endpoints of non-death patients

\begin{tabular}{lccc}
\hline Variables & Low-PNI group & High-PNI group & \\
\hline Reoperation & 4 & 1 & 0.359 \\
Yes & 36 & 39 & \\
No & $43.20 \pm 21.26$ & $44.35 \pm 21.96$ & 0.813 \\
Postoperative hospital duration (days) & $34.75 \pm 20.60$ & $34.30 \pm 22.98$ & 0.927 \\
Post-leakage hospital duration (days) & $136,421.60 \pm 77,052.49$ & $125,262.80 \pm 71,304.12$ & 0.503 \\
In-hospital cost (RMB)
\end{tabular}

$\mathrm{PNI}$, prognostic nutritional index; RMB, renminbi. 
has no significant predictive value on short-term outcomes in patients with anastomosis leakage after cancerous esophagectomy, while the in-hospital cost always tended to be lower in high-PNI group. And further studies conducted with larger cohorts were badly needed in the future to investigate significance of preoperative PNI for the patients.

\section{Acknowledgments}

Funding: This work was supported by National Natural Science Foundation of China (No. 81672291; No. 31071210) (to Yi-Dan Lin).

\section{Footnote}

Conflicts of Interest: The authors have no conflicts of interest to declare.

Ethical Statement: The authors are accountable for all aspects of the work in ensuring that questions related to the accuracy or integrity of any part of the work are appropriately investigated and resolved. The Ethics Committee of West China Hospital, Sichuan University waived the need for informed consents from those patients, while an approval for the ethical committee was obtained (approval number: 20191022).

\section{References}

1. Pennathur A, Gibson MK, Jobe BA, et al. Oesophageal carcinoma. Lancet 2013;381:400-12.

2. Mariette C, Markar SR, Dabakuyo-Yonli TS, et al. Hybrid Minimally Invasive Esophagectomy for Esophageal Cancer. N Engl J Med 2019;380:152-62.

3. Mariette C, Dahan L, Mornex F, et al. Surgery alone versus chemoradiotherapy followed by surgery for stage I and II esophageal cancer: final analysis of randomized controlled phase III trial FFCD 9901. J Clin Oncol 2014;32:2416-22.

4. van Hagen P, Hulshof MC, van Lanschot JJ, et al. Preoperative chemoradiotherapy for esophageal or junctional cancer. N Engl J Med 2012;366:2074-84.

5. Biere SS, van Berge Henegouwen MI, Maas KW, et al. Minimally invasive versus open oesophagectomy for patients with oesophageal cancer: a multicentre, open-label, randomised controlled trial. Lancet 2012;379:1887-92.

6. Ryan CE, Paniccia A, Meguid RA, et al. Transthoracic Anastomotic Leak After Esophagectomy: Current Trends.
Ann Surg Oncol 2017;24:281-90.

7. Dent B, Griffin SM, Jones R, et al. Management and outcomes of anastomotic leaks after oesophagectomy. Br J Surg 2016;103:1033-8.

8. Agzarian J, Visscher SL, Knight AW, et al. The cost burden of clinically significant esophageal anastomotic leaks-a steep price to pay. J Thorac Cardiovasc Surg 2019;157:2086-92.

9. Buzby GP, Mullen JL, Matthews DC, et al. Prognostic nutritional index in gastrointestinal surgery. Am J Surg 1980;139:160-7.

10. Hirahara N, Tajima Y, Fujii Y, et al. Preoperative Prognostic Nutritional Index Predicts Long-Term Surgical Outcomes in Patients with Esophageal Squamous Cell Carcinoma. World J Surg 2018;42:2199-208.

11. Onodera T, Goseki N, Kosaki G. Prognostic nutritional index in gastrointestinal surgery of malnourished cancer patients. Nihon Geka Gakkai Zasshi 1984;85:1001-5.

12. Lien YC, Hsieh CC, Wu YC, et al. Preoperative serum albumin level is a prognostic indicator for adenocarcinoma of the gastric cardia. J Gastrointest Surg 2004;8:1041-8.

13. Ray-Coquard I, Cropet C, Van Glabbeke M, et al. Lymphopenia as a prognostic factor for overall survival in advanced carcinomas, sarcomas, and lymphomas. Cancer Res 2009;69:5383-91.

14. Schwegler I, von Holzen A, Gutzwiller JP, et al. Nutritional risk is a clinical predictor of postoperative mortality and morbidity in surgery for colorectal cancer. Br J Surg 2010;97:92-7.

15. Nakatani M, Migita K, Matsumoto S, et al. Prognostic significance of the prognostic nutritional index in esophageal cancer patients undergoing neoadjuvant chemotherapy. Dis Esophagus 2017;30:1-7.

16. Feng JF, Chen QX. Significance of the prognostic nutritional index in patients with esophageal squamous cell carcinoma. Ther Clin Risk Manag 2014;10:1-7.

17. Nozoe T, Kimura Y, Ishida M, et al. Correlation of preoperative nutritional condition with post-operative complications in surgical treatment for oesophageal carcinoma. Eur J Surg Oncol 2002;28:396-400.

18. Filip B, Scarpa M, Cavallin F, et al. Postoperative outcome after oesophagectomy for cancer: Nutritional status is the missing ring in the current prognostic scores. Eur J Surg Oncol 2015;41:787-94.

19. Mohri $Y$, Inoue $Y$, Tanaka $K$, et al. Prognostic nutritional index predicts postoperative outcome in colorectal cancer. World J Surg 2013;37:2688-92.

20. Miyazaki T, Sakai M, Sohda M, et al. Prognostic 
Significance of Inflammatory and Nutritional Parameters in Patients with Esophageal Cancer. Anticancer Res 2016;36:6557-62.

21. Zhang $H$, Shang $X$, Ren $P$, et al. The predictive value of a preoperative systemic immune-inflammation index and prognostic nutritional index in patients with esophageal squamous cell carcinoma. J Cell Physiol 2019;234:1794-802.

22. Nakatani M, Migita K, Matsumoto S, et al. Prognostic Significance of the Prognostic Nutritional Index in Patients with Recurrent Esophageal Squamous Cell Carcinoma. Nutr Cancer 2018;70:467-73.

23. Jiang N, Deng JY, Ding XW, et al. Prognostic nutritional index predicts postoperative complications and longterm outcomes of gastric cancer. World J Gastroenterol 2014;20:10537-44.

24. Matsumoto H, Okamoto Y, Kawai A, et al. Prognosis Prediction for Postoperative Esophageal Cancer Patients Using Onodera's Prognostic Nutritional Index. Nutr Cancer 2017;69:849-54.

25. Han-Geurts IJ, Hop WC, Tran TC, et al. Nutritional status as a risk factor in esophageal surgery. Dig Surg 2006;23:159-63.

26. Okadome K, Baba Y, Yagi T, et al. Prognostic Nutritional Index, Tumor-infiltrating Lymphocytes, and Prognosis in Patients with Esophageal Cancer. Ann Surg 2018. [Epub ahead of print].

27. Sakai M, Sohda M, Miyazaki T, et al. Association of Preoperative Nutritional Status with Prognosis in Patients with Esophageal Cancer Undergoing Salvage

Cite this article as: A-Lai GH, Deng HY, Song TN, Luo J, Zhuo ZG, Shen X, Lin YD. Preoperative prognostic nutritional index shows no significant prognostic value for short-term outcomes of anastomosis-leakage patients after cancerous esophagectomy. Ann Palliat Med 2019;8(5):698-707. doi: 10.21037/apm.2019.11.08
Esophagectomy. Anticancer Res 2018;38:933-8.

28. Lee JY, Kim HI, Kim YN, et al. Clinical Significance of the Prognostic Nutritional Index for Predicting Shortand Long-Term Surgical Outcomes After Gastrectomy: A Retrospective Analysis of 7781 Gastric Cancer Patients. Medicine (Baltimore) 2016;95:e3539.

29. Kanda M, Mizuno A, Tanaka C, et al. Nutritional predictors for postoperative short-term and long-term outcomes of patients with gastric cancer. Medicine (Baltimore) 2016;95:e3781.

30. Oshi M, Kunisaki C, Miyamoto H, et al. Risk Factors for Anastomotic Leakage of Esophagojejunostomy after Laparoscopy-Assisted Total Gastrectomy for Gastric Cancer. Dig Surg 2018;35:28-34.

31. Noh GT, Ann YS, Cheong C, et al. Impact of anastomotic leakage on long-term oncologic outcome and its related factors in rectal cancer. Medicine (Baltimore) 2016;95:e4367.

32. Wen J, Bedford M, Begum R, et al. The value of inflammation based prognostic scores in patients undergoing surgical resection for oesophageal and gastric carcinoma. J Surg Oncol 2018;117:1697-707.

33. Han L, Song Q, Jia Y, et al. The clinical significance of systemic inflammation score in esophageal squamous cell carcinoma. Tumour Biol 2016;37:3081-90.

34. Chen S, Yang X, Feng JF. A novel inflammation-based prognostic score for patients with esophageal squamous cell carcinoma: the c-reactive protein/prognostic nutritional index ratio. Oncotarget 2016;7:62123-32. 